\title{
On the Peak-to-Average Power of OFDM Signals Based on Oversampling
}

\author{
Masoud Sharif, Student Member, IEEE, Mohammad Gharavi-Alkhansari, Member, IEEE, and Babak H. Khalaj
}

\begin{abstract}
Orthogonal frequency-division multiplexing (OFDM) introduces large amplitude variations in time, which can result in significant signal distortion in the presence of nonlinear amplifiers. In this paper, we introduce a new bound for the peak of the continuous envelope of an OFDM signal, based on the maximum of its corresponding oversampled sequence, that is shown to be very tight as the oversampling rate increases. The bound is then used to derive a closed-form probability upper bound for complementary cumulative distribution function of the peak-to-mean envelope power ratio of uncoded OFDM signals for sufficiently large numbers of subcarriers. As another application of the bound for oversampled sequences, we propose tight relative error bounds for computation of the peak power using two main methods: the oversampled inverse fast Fourier transform and the method introduced for coded systems based on minimum distance decoding of the code.
\end{abstract}

Index Terms-Bernstein inequality, orthogonal frequency-division multiplexing (OFDM), oversampling, peak-to-average power ratio (PAPR), peak-to-mean envelope power ratio (PMEPR).

\section{INTRODUCTION}

$\mathbf{O}$ RTHOGONAL frequency-division multiplexing (OFDM) is an attractive multicarrier modulation technique for broadband wireless access due to its strong immunity to multipath fading and high spectral efficiency. However, OFDM signals suffer from high amplitude fluctuations in time that give rise to two main issues, namely, the required large dynamic range of the quantizer in the digital section and the need for highly linear amplifiers in the analog part of the transmitter. The first problem is related to maximum value of the sampled signal, whereas, the second one is due to peak value of the continuous signal. A major part of the literature has proposed peak reduction schemes for the sampled sequence [2]-[4] or used oversampling in order to simulate the behavior of continuous signals [3], [5]-[7]. On the other hand,

Paper approved by C. Tellambura, the Editor for Modulation and Signal Design of the IEEE Communications Society. Manuscript received July 21, 2001; revised February 10, 2002; February 27, 2002; April 12, 2002; and May 20, 2002. This paper was presented in part at the IEEE International Conference on Communications, New York, NY, May 2002.

M. Sharif was with Sharif University of Technology, Tehran, Iran. He is now with the Department of Electrical Engineering, California Institute of Technology, Pasadena, CA 91125 USA (e-mail: masoud@systems.caltech.edu).

M. Gharavi-Alkhansari was with the Department of Electrical and Computer Engineering, Tarbiat Modarres University, Tehran, Iran. He is now with the Department of Electrical and Computer Engineering, McMaster University, Hamilton, ON L8S 4K1, Canada (e-mail: gharavi@ece.mcmaster.ca).

B. H. Khalaj is with the Department of Electrical Engineering, Sharif University of Technology, Tehran, Iran (e-mail: khalaj@ sharif.edu).

Digital Object Identifier 10.1109/TCOMM.2002.807619 recently, several coding schemes have been proposed to reduce the peak of the continuous signal [8]-[11]. While these codes introduce low peak power, high minimum distance, and good rates for small numbers of subcarriers $(N)$, they significantly reduce the rate of transmission for large values of $N$ [12].

The relationship between the peak of the continuous signal and the maximum of its sampled sequence has recently been addressed [2], [12], [13]. Paterson and Tarokh [12] have proposed a bound based on the Nyquist-rate sampled sequence and also for $2 \pi$ times oversampling. In this paper, we introduce a bound for oversampling rates $(k)$ greater than $\pi / \sqrt{2}$, and we show that the bound is accurate as $k$ increases. Then, we use this new perspective as a cornerstone of our study in two parts. In the first part, we introduce a closed-form probability upper bound for the complementary cumulative distribution function (CCDF) of the peak-to-mean envelope power ratio (PMEPR) of uncoded OFDM signals for sufficiently large values of $N$. As an immediate consequence of the probability bound, we show that asymptotically, there exist codes with high rate and PMEPR of less than $2 \ln N+1 / 4$.

In the second part, we consider methods to compute the peak power of OFDM signals for both coded and uncoded systems. Computation of the peak power plays a major role in peak reduction methods that optimize the peak power over free parameters, such as initial phases in conjunction with coding [1] and partial transmit sequence (PTS) [4], [6]. Recently, Tarokh and Jafarkhani [1] have introduced an efficient peak value computation method along with its corresponding error bound for coded OFDM systems. On the other hand, oversampled inverse fast Fourier transform (IFFT) has been traditionally used in simulations in order to compute the envelope peak of OFDM signals [3], [5]. In this paper, we introduce new relative error bounds for the above two methods. Our results not only provide an analytic relationship between $k$ and estimation accuracy but also result in much tighter bounds compared to the earlier results of [1] and [13].

This paper is organized as follows. In Section II, we define the peak factors used to quantify the amplitude fluctuation of the signals. Section III introduces a bound to relate the peak of the continuous signal to the maximum of its oversampled sequence. Based on this bound, Section IV introduces a probability upper bound for the CCDF of PMEPR and investigates its implication on the rate of codes with bounded PMEPR. Section V presents tight relative error bounds in computation of the peak power. Finally, Section VI concludes the paper. 


\section{DEFINITIONS}

The complex envelope of a band-limited OFDM signal with $N$ subcarriers may be approximated as [1]

$$
s(t)=\sum_{n=0}^{N-1} c_{n} e^{j 2 \pi n f_{0} t}, \quad 0 \leq t<T
$$

and consequently, the OFDM signal is given by

$$
G(t)=\operatorname{Re}\left\{\sum_{n=0}^{N-1} c_{n} e^{j 2 \pi\left(f_{c}+n f_{0}\right) t}\right\}, \quad 0 \leq t<T
$$

where $f_{0}$ is the subchannel spacing, $f_{c}$ is the carrier frequency, $T$ is the symbol period, and $\bar{c}=\left(c_{0}, \ldots, c_{N-1}\right)$ is a vector of complex symbols from a given $q$-ary constellation. The admissible vectors are called codewords, where the ensemble of all possible codewords constitutes the code $C$. Clearly, in an uncoded system, all $c_{i}$ 's are chosen uniformly and independently from the $q$-ary constellation, and so $C$ is the set of all $q^{N}$ possible codewords for uncoded systems. Since the cyclic prefix cannot introduce any new peaks in the symbol, we assume that $f_{0}=1 / T$. Also, for mathematical convenience, we substitute $\theta=2 \pi t / T$ to get

$$
s(\theta)=\sum_{n=0}^{N-1} c_{n} e^{j n \theta}, \quad 0 \leq \theta<2 \pi .
$$

The level of amplitude fluctuation of OFDM signals is usually measured in terms of peak factors that indicate the ratio of the peak power to the average envelope power of the signal. More specifically, peak-to-average power ratio (PAPR) of the transmit signal is defined as [1]

$$
\operatorname{PAPR}(\xi)=\max _{C, 0 \leq \theta<2 \pi} \frac{\left|\operatorname{Re}\left\{\sum_{n=0}^{N-1} c_{n} e^{j(\xi+n) \theta}\right\}\right|^{2}}{P_{\mathrm{av}}}
$$

where $\xi$ represents the normalized carrier frequency $f_{c} / f_{0}$ and $P_{\mathrm{av}}$ is a constant that depends on the code family $P_{\mathrm{av}}=E\left\{\|\bar{c}\|^{2}\right\}$. Similarly, PMEPR is defined as [12], [13]

$$
\text { PMEPR }=\max _{C, 0 \leq \theta<2 \pi} \frac{\left|\sum_{n=0}^{N-1} c_{n} e^{j n \theta}\right|^{2}}{P_{\mathrm{av}}} .
$$

Obviously, PAPR measures the peak of the signal at the analog front end, but PMEPR can be used both as a peak of the baseband signal and as an upper bound for the peak in the transmitter front end. In Section V, the relationship between PAPR and PMEPR will be discussed.

\section{MAXimum of THE OVERSAMPLED OFDM Signal}

It was recently shown that the peak of a continuous OFDM signal can be bounded by the maximum of its oversampled sequence [12], [13]. In this section, we initially propose an even tighter bound for oversampling rates greater than $\pi / \sqrt{2}$, and then we discuss the tightness of the bound. We first prove the following fundamental theorem.

Theorem 1: Let $s(\theta)$ be as defined in (3), and assume that $\left|s\left(\theta_{0}\right)\right|=\max _{0 \leq \theta<2 \pi}|s(\theta)|$. Then for any real value of $\beta>0$ and for all $\theta$ in the interval $\left[\theta_{0}-1 / N \sqrt{(2 \beta) /(\beta+1)}, \theta_{0}+\right.$ $1 / N \sqrt{(2 \beta) /(\beta+1)}]$, we have

$$
|s(\theta)|>\sqrt{\frac{1}{\beta+1}}\left|s\left(\theta_{0}\right)\right| .
$$

Proof: We may define $p(\theta)$ as

$$
p(\theta)=|s(\theta)|^{2}=\sum_{n=0}^{N-1} a_{n} \cos n \theta+b_{n} \sin n \theta
$$

where $a_{n}$ and $b_{n}$ are real numbers related to all $c_{n}$ 's. Since $p(\theta)$ is maximized at $\theta_{0}, p^{\prime}\left(\theta_{0}\right)=0$, we may write the second-order Taylor expansion of $p(\theta)$ around the point $\theta_{0}$ as

$$
p(\theta)=p\left(\theta_{0}\right)+\frac{1}{2}\left(\theta-\theta_{0}\right)^{2} p^{\prime \prime}\left(\theta^{*}\right)
$$

where $\theta^{*}$ is a point between $\theta_{0}$ and $\theta$. Taking the absolute value from both sides, using the triangle inequality, and noting that $p(\theta) \geq 0$, we get

$$
p(\theta) \geq p\left(\theta_{0}\right)-\frac{1}{2}\left(\theta-\theta_{0}\right)^{2}\left|p^{\prime \prime}\left(\theta^{*}\right)\right| .
$$

Using the classic inequality of [14], for any polynomial $p(\theta)$ with the form of (7) with real coefficients $a_{n}$ and $b_{n}$, we have $\max _{0 \leq \theta<2 \pi}\left|p^{(r)}(\theta)\right| \leq(N-1)^{r} \max _{0 \leq \theta<2 \pi}|p(\theta)|$. For $r=2$, this becomes

$$
\max _{0 \leq \theta<2 \pi}\left|p^{\prime \prime}(\theta)\right|<N^{2} \max _{0 \leq \theta<2 \pi}|p(\theta)| .
$$

Combining (9) and (10) gives

$$
\begin{aligned}
|s(\theta)|^{2}=p(\theta) & >\left(1-\frac{N^{2}}{2}\left(\theta-\theta_{0}\right)^{2}\right) p\left(\theta_{0}\right) \\
& =\left(1-\frac{N^{2}}{2}\left(\theta-\theta_{0}\right)^{2}\right)\left|s\left(\theta_{0}\right)\right|^{2} .
\end{aligned}
$$

Therefore, by comparing (6) and (11) and choosing the appropriate value of $\beta$, we get $|s(\theta)|>\sqrt{1 /(\beta+1)}\left|s\left(\theta_{0}\right)\right|$, for the values of $\theta$ in the interval of $\left[\theta_{0}-1 / N \sqrt{(2 \beta) /(\beta+1)}, \theta_{0}+\right.$ $1 / N \sqrt{(2 \beta) /(\beta+1)}]$.

Theorem 2: Let $s(\theta)$ be as defined in Theorem 1, then for any $k$ greater than $\pi / \sqrt{2}$, the maximum of $|s(\theta)|$ is bounded by maximum of its $k N$ samples on the unit circle by

$$
\max _{0 \leq \theta<2 \pi}|s(\theta)|<\sqrt{\frac{k^{2}}{k^{2}-\pi^{2} / 2}} \max _{1 \leq p \leq k N} \mid s\left(\frac{2 \pi p}{k N}\right) .
$$

Proof: Following the same statement as in Theorem 1, let $e^{j \theta_{0}}$ be the point on the unit circle for which $\max _{0 \leq \theta<2 \pi}|s(\theta)|=$ $\left|s\left(\theta_{0}\right)\right|$. Also assume $e^{j \theta_{p}}$ to be the primitive $k N$ roots of unity defined as

$$
\theta_{p}=\frac{2 \pi p}{k N}, \quad p \in\{1,2, \ldots, k N\} .
$$

Obviously, there exists a $p$ such that $\left|\theta_{0}-\theta_{p}\right| \leq(2 \pi) /(2 k N)=$ $\pi /(k N)$, therefore, using (11), we get

$$
\left|s\left(\theta_{p}\right)\right|^{2}>\left(1-\frac{N^{2}}{2}\left(\frac{\pi}{k N}\right)^{2}\right)\left|s\left(\theta_{0}\right)\right|^{2} .
$$

Consequently, rearranging (14) results in the following nontrivial inequality for $k>\pi / \sqrt{2}$ :

$$
\max _{0 \leq \theta<2 \pi}|s(\theta)|<\sqrt{\frac{k^{2}}{k^{2}-\pi^{2} / 2}}\left|s\left(\theta_{p}\right)\right|
$$

and maximizing over $p$, completes the proof and leads to (12). 


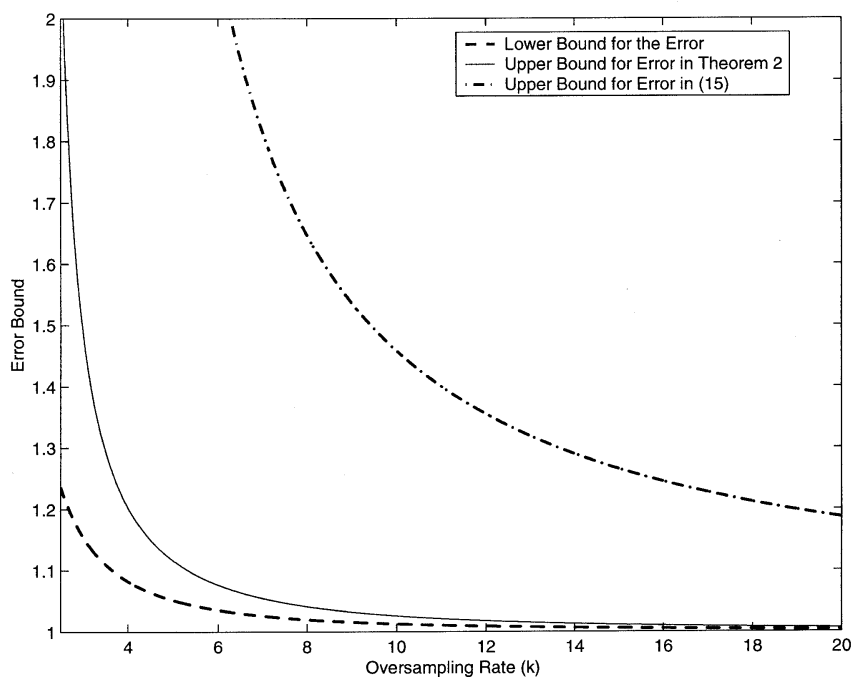

Fig. 1. Error obtained for $1+e^{j(N-1) \theta}$ in comparison with the upper bound in (12) and (15) as a function of oversampling rate.

Remark 1: It is worth mentioning that by using the firstorder Taylor expansion of $p(\theta)$ around the point $\theta_{0}$ and using Bernstein inequality, we get

$$
\begin{aligned}
|s(\theta)|^{2}=p(\theta) & >\left(1-N\left(\theta-\theta_{0}\right)\right) p\left(\theta_{0}\right) \\
& =\left(1-N\left(\theta-\theta_{0}\right)\right)\left|s\left(\theta_{0}\right)\right|^{2} .
\end{aligned}
$$

By following the same statement as in Theorem 2, we get

$$
\max _{0 \leq \theta<2 \pi}|s(\theta)|<\sqrt{\frac{k}{k-\pi}} \max _{1 \leq p \leq k N}\left|s\left(\frac{2 \pi p}{k N}\right)\right| .
$$

The improvement from (15) to (12) may suggest that using higher order Taylor expansions and Bernstein inequality may result in finding better bounds. Unfortunately, without using any additional information about the derivatives of $p(\theta)$, this approach does not seem to further improve the bound in (12).

In order to investigate the tightness of the bound in (12), we may define the error in estimating the maximum of $s(\theta)$ by its $k N$ samples as $\chi=\max _{0 \leq \theta \leq 2 \pi}|s(\theta)| / \max _{1 \leq p \leq k N}\left|s\left(\theta_{p}\right)\right|$. A lower bound on $\chi$ can be found by considering $s_{0}(\theta)=1+e^{j(N-1) \theta}$ and its samples at $\theta_{p}=(2 p-1) \pi /(k N)$, then clearly, $\chi \geq$ $1 / \cos (\pi(N-1) /(2 k N)) \approx 1 / \cos (\pi / 2 k)$ for large values of $N$. Fig. 1 compares the upper bounds on $\chi$ in (12) and (15) with its lower bound. Fig. 1 implies that the bound in (12) is accurate as $k$ increases. As an example, when $k$ equals 4 and 16 , the bound in (12) suggests maximum differences of $1.6 \mathrm{~dB}$ and $0.08 \mathrm{~dB}$, respectively.

\section{PRoBABILITY Bound ON PMEPR}

Recently, the probability distribution of PMEPR has been used to evaluate the performance of PMEPR reduction schemes [13], [15], [17]. For large values of $N$ or large constellation size, the computational effort in finding PMEPR will become prohibitively large [17]. In this section, we first find an upper bound for the CCDF of PMEPR for uncoded OFDM signals. Then, we use this bound to investigate the rate of a code constructed by removing the codewords with high PMEPR from the set of all codewords in the uncoded system.
In order to find the probability bound, Theorem 2 can be used to convert the maximization over the continuous variable $\theta$ in (5) to a discrete form. We can then solve the discrete problem by assuming a Gaussian distribution for each sample of $s(\theta)$, which is valid by the virtue of the central limit theorem for large values of $N$ and uncoded OFDM signals.

It is worth noting that the distribution of PMEPR has been addressed in [17] by numerically computing the distribution for small values of $N$ (i.e., $N \leq 15$ ) and also a lower bound on $\operatorname{Pr}\{\mathrm{PMEPR}>\lambda\}$ is introduced by considering the maximum of $N$ samples of the signal and assuming that the $N$ samples have jointly Gaussian distribution which is not mathematically rigorous. On the other hand, in [15], PMEPR distribution has been derived under the strong assumption that the OFDM signals behave as a band-limited Gaussian process. However, we will only use the Gaussian assumption for each sample of the OFDM signal, and there is no further assumption on the joint distribution of samples.

Theorem 3: Let $C$ be the set of all codewords in an uncoded system, then, for every $k>\pi / \sqrt{2}$ such that $k N$ is an integer, and for sufficiently large values of $N$, we have

$$
\operatorname{Pr}\{\mathrm{PMEPR}>\lambda\}<k N e^{-\lambda\left(1-\frac{\pi^{2}}{2 k^{2}}\right)} .
$$

Proof: Using Theorem 2, for every $k>\pi / \sqrt{2}$ such that $k N$ is an integer, we may write

$\operatorname{Pr}\{\operatorname{PMEPR}>\lambda\}<\operatorname{Pr}\left\{\max _{1 \leq p \leq k N} \frac{\left|s\left(\theta_{p}\right)\right|^{2}}{P_{\mathrm{av}}} \lambda\left(1-\frac{\pi^{2}}{2 k^{2}}\right)\right\}$

where $\theta_{p}$ is as defined in (13). Considering that all $c_{i}$ 's are independently and uniformly distributed in an uncoded system, for sufficiently large $N, s\left(\theta_{p}\right)$ can be considered as a complex Gaussian random variable with variance $P_{\text {av }}$ [16]. Therefore, $\left|s\left(\theta_{p}\right)\right|$ has the Rayleigh distribution, and by using the union bound to calculate (17), we get

$$
\begin{aligned}
\operatorname{Pr}\left\{\max _{1 \leq p \leq k N}\right. & \left.\frac{\left|s\left(\theta_{p}\right)\right|^{2}}{P_{\mathrm{av}}}>\lambda\left(1-\frac{\pi^{2}}{2 k^{2}}\right)\right\} \\
& =\operatorname{Pr}\left\{\exists i \in\{1, \ldots, k N\}: \frac{\left|s\left(\theta_{i}\right)\right|^{2}}{P_{\mathrm{av}}}>\lambda\left(1-\frac{\pi^{2}}{2 k^{2}}\right)\right\} \\
& \leq k N \operatorname{Pr}\left\{\frac{|s(\theta)|^{2}}{P_{\mathrm{av}}}>\lambda\left(1-\frac{\pi^{2}}{2 k^{2}}\right)\right\} \\
& =k N e^{-\lambda\left(1-\frac{\pi^{2}}{2 k^{2}}\right)} .
\end{aligned}
$$

The theorem follows immediately from (17) and (18).

Since the bound is valid for every $k>\pi / \sqrt{2}$, we can tighten the bound over $k$ and find the optimal $k$ by differentiating (16) with respect to $k$. The resulting optimum oversampling rate, $k_{\text {opt }}$, will be given as

$$
\frac{\pi^{2}}{k_{\mathrm{opt}}^{2}}\left(1-\frac{\pi^{2}}{2 k_{\mathrm{opt}}^{2}}\right)=\frac{1}{2 \lambda} .
$$

Apparently, $k_{\mathrm{opt}} N$ should be an integer. Fig. 2 shows the upper bound with $k_{\mathrm{opt}}$ is chosen for each value of $\lambda$ from (16), and the simulation results for $\operatorname{Pr}\{\mathrm{PMEPR}>\lambda\}$ with $N=128$, a quarternary phase-shift keying (QPSK) constellation, and $k=$ 16.

Corollary 1: Let $C$ be the set of all codewords in an uncoded system, in which all $c_{i}$ 's are chosen from a given constellation with average and maximum energy $E_{\mathrm{av}}$ and 


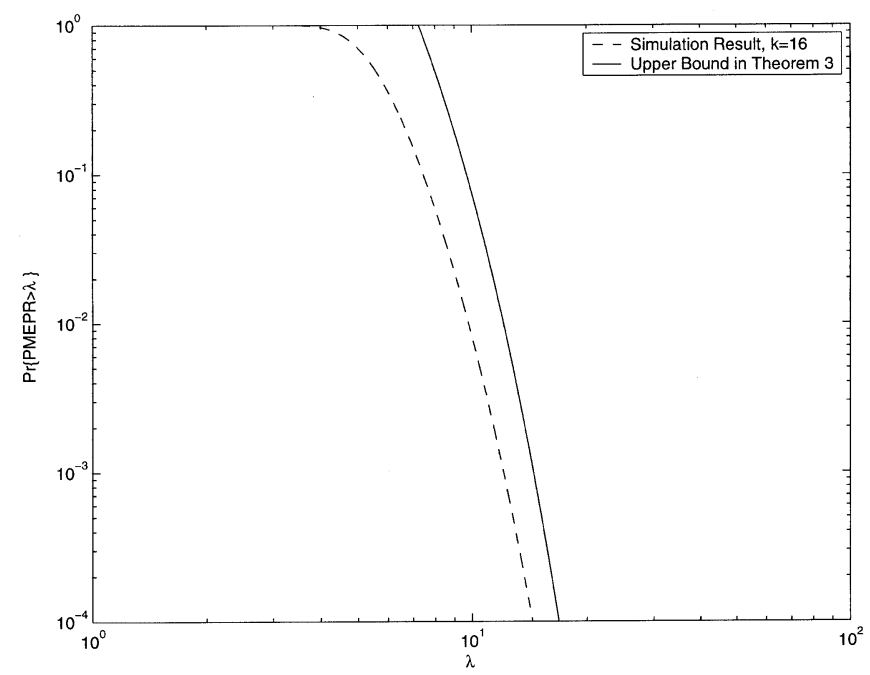

Fig. 2. Upper bound and simulation result for CCDF of PMEPR for $N=128$ and QPSK constellation.

$E_{\max }$, respectively. Let $C^{\prime}$ be the set of codewords in $C$ such that $\max _{0 \leq \theta<2 \pi}|s(\theta)|^{2}<(2 \ln N+1 / 4) N E_{\mathrm{av}}$, then asymptotically removing codewords with high PMEPR does not have a catastrophic impact on the number of codewords. In particular, asymptotically $\lim _{N \rightarrow \infty}\left|C^{\prime}\right| /|C|=1$ and $\operatorname{PMEPR}\left(C^{\prime}\right)<2 \ln N+1 / 4$.

Proof: Using Theorem 3 and considering $k=\lfloor\pi \sqrt{2 \lambda}\rfloor$, which is asymptotically optimal for large values of $\lambda$, we can find a lower bound for the number of codewords in $C$ with $\max _{0 \leq \theta<2 \pi}|s(\theta)|^{2}<(2 \ln N+1 / 4) N E_{\text {av }}$ as

$$
\begin{aligned}
|C| \geq\left|C^{\prime}\right| & =|C| \operatorname{Pr}\{\text { PMEPR }<2 \ln N+1 / 4\} \\
& \geq|C|\left(1-\frac{\lfloor\pi \sqrt{2(2 \ln N+1 / 4)}\rfloor}{N}\right)
\end{aligned}
$$

equivalently, $\left(|C|-\left|C^{\prime}\right|\right) /|C| \leq O(\sqrt{\ln N} / N)$, which shows that $\lim _{N \rightarrow \infty}\left|C^{\prime}\right| /|C|=1$. In order to find $\operatorname{PMEPR}\left(C^{\prime}\right)$, we can simply find a lower bound for $P_{\mathrm{av}}\left(C^{\prime}\right)$ by considering the maximum power for all deleted codewords. Therefore

$$
\begin{aligned}
P_{\mathrm{av}}\left(C^{\prime}\right) & \geq \frac{N E_{\mathrm{av}}|C|-N E_{\max }\left(|C|-\left|C^{\prime}\right|\right)}{\left|C^{\prime}\right|} \\
& >\frac{N E_{\mathrm{av}}|C|-N E_{\max }\left(|C|-\left|C^{\prime}\right|\right)}{|C|} \\
& \geq N E_{\mathrm{av}}\left(1-\frac{E_{\mathrm{max}}}{E_{\mathrm{av}}} O\left(\frac{\sqrt{\ln N}}{N}\right)\right)
\end{aligned}
$$

which shows that asymptotically $P_{\text {av }}\left(C^{\prime}\right) \geq N E_{\text {av }}$. Considering that all the codewords in $C^{\prime}$ have maximum envelope power of less than $N E_{\mathrm{av}}(2 \ln N+1 / 4)$, we can use the definition of PMEPR in (5) to get $\operatorname{PMEPR}\left(C^{\prime}\right)<2 \ln N+1 / 4$.

Although the result of this corollary seems to be promising, currently no practically implementable method is known for performing this process of removing bad codewords from a code family, and existing codes with low PMEPR have a very low rate asymptotically [11], [12].

It is worth mentioning that in [12], it is proved that the Varsharmov-Gilbert region for minimum distance and rate of spherical codes with PMEPR less than $8 \ln N$ is the same as the region for codes with unconstrained PMEPR. Similarly, Corollary 1 states that almost all the $q$-ary codewords in the uncoded set have PMEPR of less than $2 \ln N+1 / 4$.

\section{PMEPR AND PAPR COMPUTATION}

There are several methods to reduce the peak factors by optimizing PMEPR or PAPR over free parameters, such as using optimum phases in the PTS method [4], [6], [7], and initial phases in conjunction with coding [1]. In order to optimize PMEPR, oversampled IFFT of the modulating vector has been used to compute PMEPR. On the other hand, Tarokh and Jafarkhani [1] have recently introduced an efficient method, along with its corresponding error bound, for PAPR computation in coded OFDM systems. In this section, we introduce new relative error bounds for the above computation methods.

\section{A. IFFT Method}

Intuitively, maximum of the oversampled IFFT of the modulating vector can provide a good estimate for the peak of the continuous modulated signal. In the following theorem, by using the result of Theorem 2, the relative error bound of [13] will be significantly improved.

Theorem 4: Let $C$ be a code family and $s(\theta)$ be as defined in (3). Then, PMEPR of $C$ can be estimated by using $k N$-point IFFT of each codeword and maximizing over all the codewords in $C$ as

$$
\overline{\mathrm{PMEPR}}=\max _{1 \leq p \leq k N, C} \frac{|s(2 \pi p / k N)|^{2}}{P_{\mathrm{av}}}
$$

then, the relative error for $k>\pi / \sqrt{2}$ is bounded by

$$
0 \leq \frac{\text { PMEPR }-\overline{\text { PMEPR }}}{\overline{\text { PMEPR }}}<\frac{\pi^{2} / 2}{k^{2}-\pi^{2} / 2} .
$$

Proof: Using Theorem 2, the continuous problem of PMEPR computation can be converted to discrete form, and by maximizing both sides of (12) over all the codewords, we may write

$\max _{0 \leq \theta<2 \pi, C}\left|\sum_{n=0}^{N-1} c_{n} e^{j n \theta}\right|^{2}<\frac{k^{2}}{k^{2}-\pi^{2} / 2} \max _{1 \leq p \leq k N, C}\left|\sum_{n=0}^{N-1} c_{n} e^{j \frac{2 \pi n p}{k N}}\right|^{2}$

for values of $k$ greater than $\pi / \sqrt{2}$. Normalizing both sides of (22) to the average power, we get PMEPR < $k^{2} \overline{\mathrm{PMEPR}} /\left(k^{2}-\pi^{2} / 2\right)$, which directly leads to (21).

Theorem 4 shows that for large values of $k$, the relative estimation error is inversely proportional to the square of $k$ rather than $k$, as proposed in [13]. 


\section{B. Method of Tarokh and Jafarkhani}

Recently, in [1], a novel PAPR computation method has been introduced which is based on maximum-likelihood decoding of the code $C$. In this approach, the estimated value is defined as

$$
\begin{aligned}
G_{L}(\xi)= & \max _{\theta_{i} \in\left\{, \theta_{1}, \ldots, \theta_{L}\right\}} \\
& \left\{\max _{\bar{c} \in C} \frac{\left.\operatorname{Re}\left\{\sum_{n=0}^{N-1} c_{n} e^{j(\xi+n) \theta_{i}}\right\}\right|^{2}}{P_{\mathrm{av}}}\right\}
\end{aligned}
$$

where the inner maximization is computed for $L$ time samples using the efficient method of [1]. Moreover, arbitrary values of $\xi_{1}$ satisfying $\xi_{1} \leq \xi$ may be used in $G_{L}$ instead of $\xi$ in order to estimate $\operatorname{PAPR}(\xi)$. By using this method, the error in estimating $\operatorname{PAPR}(\xi)$ can be bounded as [1]

$$
\left|\operatorname{PAPR}(\xi)-G_{L}\left(\xi_{1}\right)\right| \leq\left\{\frac{2 \pi N\left(N+\xi_{1}\right)}{L}+\frac{2 \pi N^{2}}{\sqrt{3} \xi_{1}}\right\} Q
$$

where $Q$ equals the ratio of the maximum energy to the average energy of the constellation.

It should be noted that (24) predicts a high number of time samples is required to achieve reasonable estimation error levels. However, as mentioned in [1], the upper bound for the absolute value of error is not tight, and simulation results show a much higher accuracy. In what follows, we derive a tight relative error bound for the estimation of PAPR $(\xi)$ and PMEPR by computing $G_{L}\left(\xi_{1}\right)$ at integer values of $\xi_{1}$.

Lemma 5: Let $k>\pi / \sqrt{2}$, and $r$ and $L=2(N+r) k$ be two positive integers, then

$$
G_{L}(r) \leq \operatorname{PAPR}(r)<\frac{k^{2}}{k^{2}-\pi^{2} / 2} G_{L}(r) .
$$

Proof: Let $h(\theta)=\sum_{n=0}^{N-1} c_{n} e^{j(n+r) \theta}$, and $h_{e}(\theta)=$ $\sum_{n=0}^{N-1} c_{n} e^{j n \theta}$, where $h_{e}(\theta)$ is the complex baseband envelope of $h(\theta)$. Therefore, we may write $\operatorname{Re}\{h(\theta)\}=$ $\left|h_{e}(\theta)\right| \cos \left(r \theta+\angle h_{e}(\theta)\right)$. In order to bound the PAPR with its sampled sequence, we may write $\operatorname{Re}^{2}\{h(\theta)\}$ as

$$
\begin{aligned}
\operatorname{Re}^{2}\{h(\theta)\}= & \cos ^{2}(r \theta) \operatorname{Re}^{2}\left\{h_{e}(\theta)\right\} \\
& +\sin ^{2}(r \theta) \operatorname{Im}^{2}\left\{h_{e}(\theta)\right\} \\
& -\sin (2 r \theta) \operatorname{Re}\left\{h_{e}(\theta)\right\} \operatorname{Im}\left\{h_{e}(\theta)\right\} \\
= & \sum_{n=o}^{2(N+r-1)} \alpha_{n} \cos (n \theta)+\beta_{n} \sin (n \theta) .
\end{aligned}
$$

Since (26) has the same form as (7), Lemma 5 immediately follows by the application of Theorem 2 and noticing that $L=$ $2(N+r) k$ samples give higher accuracy than $L=2(N+r) k-$ 1.

In the next theorem, we develop a new relationship between PAPR $(r)$ and PMEPR at integer values of $r$ and only for the case of $N\langle\langle r$.
Theorem 6: Let $k, L$, and $r$ be as defined in Lemma 5. If $N\left\langle\left\langle r\right.\right.$ and $e^{j 2 \pi N / r}$ can be approximated by 1 , then

$$
\operatorname{PMEPR} \geq \operatorname{PAPR}(r)>\left(1-\frac{\pi^{2} N^{2}}{2 r^{2}}\right) \operatorname{PMEPR} .
$$

Proof: Let $h(\theta)$ and $h_{e}(\theta)$ be as defined in Lemma 5. Also assume that PMEPR (or, equivalently, $\left|h_{e}(\theta)\right|$ ) reaches its maximum value at phase $\alpha_{0}$ and with the codeword $\bar{c}$. First, we find the distance between two neighboring points $\alpha_{1}$ and $\alpha_{2}$ in $[0,2 \pi)$, selected such that

$$
h\left(\alpha_{1}\right)=h\left(\alpha_{2}\right) \text { and } \angle h\left(\alpha_{1}\right)=\angle h\left(\alpha_{2}\right)=0 .
$$

Since the carrier frequency is much greater than the highest frequency of the baseband signal, $h(\theta)$ can attain any phase value in $[0,2 \pi)$ by a small change in $\theta$ [1]. Therefore, there are several phases such as $\alpha_{1}$, where the phase of the complex signal $h\left(\alpha_{1}\right)$ is zero. We can now prove that $\alpha_{2}=\alpha_{1}+2 \pi / r$ satisfies (28), which may be written as

$$
\begin{aligned}
h\left(\alpha_{2}\right) & =h\left(\alpha_{1}+2 \pi / r\right) \\
& =e^{j 2 \pi} \sum_{n=0}^{N=1} c_{n} e^{j(r+n) \alpha_{1}} e^{j 2 \pi n / r} .
\end{aligned}
$$

As $N\left\langle\left\langle r\right.\right.$, we may use the approximation $e^{j 2 \pi n / r} \approx 1$ for $n \in$ $\{0,1, \ldots, N-1\}$ in (29) in order to obtain (28). Considering the zero phase of $h(\theta)$ at $\alpha_{1}$ and $\alpha_{2}$, for $i=1,2$, we get

$$
\begin{aligned}
\left|\sum_{n=0}^{N=1} c_{n} e^{j(n+r) \alpha_{i}}\right| & =\operatorname{Re}\left\{\sum_{n=0}^{N-1} c_{n} e^{j(n+r) \alpha_{i}}\right\} \\
& =\left|\sum_{n=0}^{N-1} c_{n} e^{j n \alpha_{i}}\right| .
\end{aligned}
$$

Now consider the two neighboring points $\gamma_{1}$ and $\gamma_{2}$ such $\gamma_{2}=\gamma_{1}+2 \pi / r$ and $\gamma_{2}>\alpha_{0} \geq \gamma_{1}$, for which $h(\theta)$ satisfies (28). From (30), we have

$$
\operatorname{Re}\left\{\sum_{n=0}^{N-1} c_{n} e^{j(n+r) \gamma_{i}}\right\}=\left|\sum_{n=0}^{N-1} c_{n} e^{j n \gamma_{i}}\right| .
$$

Applying Theorem 1 to $h_{e}(\theta)$, we can assign the minimum width of the peak of $h_{e}(\theta)$ in the interval $\left[\gamma_{1}, \gamma_{2}\right]$ to be equal to $2 \pi / r$, and then find the corresponding threshold constant $(\beta)$ in Theorem 1. Therefore, $2 / N \sqrt{(2 \beta) /(\beta+1)}=2 \pi / r$ and then, Theorem 1 implies that

$$
\left|\sum_{n=0}^{N-1} c_{n} e^{j n \theta}\right|>\sqrt{1-\frac{\pi^{2} N^{2}}{2 r^{2}}} \times \max _{0 \leq \theta<2 \pi}\left|\sum_{n=0}^{N-1} c_{n} e^{j n \theta}\right| \theta \in\left[\gamma_{1}, \gamma_{2}\right] .
$$

Evaluating both sides of (32) at $\gamma_{1}$ and $\gamma_{2}$, dividing them by $P_{\mathrm{av}}$, and using (31), we get

$$
\operatorname{PAPR}\left(\gamma_{1} \text { and } \gamma_{2}\right)>\left(1-\frac{\pi^{2} N^{2}}{2 r^{2}}\right) \operatorname{PMEPR} \text {. }
$$


TABLE I

ERROR BOUND OF [1] COMPARED WITH RESULT OF THEOREM 7 , WHERE $N$ IS THE NUMBER OF SUBCARRIERS

\begin{tabular}{c|c|c|c}
\hline$r$ & $L$ & $\begin{array}{c}\text { Error Bound of [1] } \\
\text { for Computation of } \\
\text { PAPR }(r)\end{array}$ & $\begin{array}{c}\text { Relative Error Bound of } \\
\text { (34) for Computation of } \\
\text { PMEPR and PAPR }(r)\end{array}$ \\
\hline$N^{2}$ & $2\left(N^{2}+N^{3}\right)$ & $6.7 Q$ & $\pi^{2} / 2 N^{2}+\pi^{2} /\left(2 N^{2}-\pi^{2}\right)$ \\
\hline$N^{3}$ & $2\left(N^{3}+N^{5}\right)$ & $6.7 Q / N$ & $\pi^{2} / 2 N^{4}+\pi^{2} /\left(2 N^{4}-\pi^{2}\right)$ \\
\hline$N^{4}$ & $2\left(N^{4}+N^{7}\right)$ & $6.7 Q / N^{2}$ & $\pi^{2} / 2 N^{6}+\pi^{2} /\left(2 N^{6}-\pi^{2}\right)$ \\
\hline
\end{tabular}

On the other hand, it is obvious that $\operatorname{PAPR}\left(\gamma_{1}, \gamma_{2}\right) \leq$ $\operatorname{PAPR}(r) \leq \mathrm{PMEPR}$. Therefore, Theorem 6 follows directly from (33).

Theorem 7: Let $r, L$, and $k$ be as defined in Lemma 5, and $N\langle\langle r$ as in Theorem 6. Then, the relative error in estimating PAPR $(\xi)$ and PMEPR by $G_{L}(r)$ will be given by

$$
\begin{aligned}
\left|1-\frac{G_{L}(r)}{\operatorname{PAPR}(\xi)}\right| & \leq\left|1-\frac{G_{L}(r)}{\operatorname{PMEPR}}\right| \\
& <\frac{\pi^{2} N^{2}}{2 r^{2}}+\frac{\pi^{2} / 2}{k^{2}-\pi^{2} / 2} .
\end{aligned}
$$

Proof: Using Theorem 6, we may write

$\frac{|\mathrm{PMEPR}-\mathrm{PAPR}(r)|}{\mathrm{PMEPR}}<1-\left(1-\frac{\pi^{2} N^{2}}{2 r^{2}}\right)=\frac{\pi^{2} N^{2}}{2 r^{2}}$

By using $G_{L}(r) \leq$ PMEPR, we can rewrite (25) as

$$
\frac{\left|\operatorname{PAPR}(r)-G_{L}(r)\right|}{\operatorname{PMEPR}}<\left(\frac{k^{2}}{k^{2}-\pi^{2} / 2}\right)-1=\frac{\pi^{2} / 2}{k^{2}-\pi^{2} / 2} .
$$

Using the triangle inequality, (35), and (36), we can prove the right inequality in (34). Since $\operatorname{PAPR}(\xi) \leq \mathrm{PMEPR}$, the left-hand side of (34) is evident as well.

Table I shows the bound for absolute value of error in [1], compared with the relative error bound derived in Theorem 7 for computation of PMEPR and PAPR $(r)$ in an OFDM system with $N$ subcarriers. It is evident from Table I that the relative error bound of (34), in the worst case, is much tighter than the bound of [1] as given in (24). For example, for the case of $L=2\left(N^{2}+N^{3}\right)$ and $N=48$ considered in [1], our proposed relative error is smaller than 0.0043 . Moreover, our relative error bound applies to both PMEPR and PAPR computation.

\section{CONCLUSION}

In this paper, a bound for the peak of the continuous envelope of OFDM signals was proposed based on the maximum of its corresponding oversampled sequence. It was shown that as the oversampling rate increases, we can tightly bound the continuous envelope by its oversampled sequence. Two problems were then addressed. First, a closed-form upper bound for the CCDF of PMEPR of an uncoded system was derived and an implication of the probability bound on the rate of codes with bounded PMEPR was presented. Secondly, computation methods of PMEPR and PAPR, using oversampled IFFT and also the method proposed in [1], were considered and tight relative estimation errors as a function of oversampling rate for both methods were derived.

\section{ACKNOWLEDGMENT}

The authors are indebted to C. Tellambura for his comment on Theorem 1 that improved the bound and changed the theorem to its present form. Thanks are also due to the anonymous reviewers for their constructive suggestions.

\section{REFERENCES}

[1] V. Tarokh and H. Jafarkhani, "On the computation and reduction of the peak to average power ratio in multicarrier communications," IEEE Trans. Commun., vol. 48, pp. 37-44, Jan. 2000.

[2] D. Wulich, "Comments on the peak factor of sampled and continuous signals," IEEE Commun. Lett., vol. 4, pp. 213-214, July 2000.

[3] C. Tellambura, "Use of m-sequence for OFDM peak-to-average power ratio reduction," Electron. Lett., vol. 33, no. 15, pp. 1300-1301, July 1997.

[4] S. H. Müller and J. B. Huber, "A comparison of peak power reduction schemes for OFDM," in Proc. Global Communications Conf., vol. 1, 1997, pp. 1-5.

[5] X. Li and L. J. Cimini, "Effects of clipping and filtering on the performance of OFDM," in Proc. IEEE Vehicular Technology Conf., May 1997, pp. 1634-1638.

[6] C. Tellambura, "Improved phase factor computation for the PAR reduction of an OFDM signal using PTS," IEEE Commun. Lett., vol. 5, pp. 135-137, Apr. 2001.

[7] _ - "Computation of the continuous-time PAR of an OFDM signal with BPSK subcarriers," IEEE Commun. Lett., vol. 5, pp. 185-187, May 2001.

[8] J. A. Davis and J. Jedwab, "Peak to mean power control in OFDM, Golay complementary sequences and Reed-Muller codes," IEEE Trans. Inform. Theory, vol. 45, pp. 2397-2417, Nov. 1999.

[9] K. G. Paterson, "Generalized Reed-Muller codes and power control in OFDM modulation," IEEE Trans. Inform. Theory, vol. 46, pp. 104-120, Jan. 2000.

[10] C. Roessing and V. Tarokh, "A construction of OFDM 16-QAM sequences having low peak powers," IEEE Trans. Inform. Theory, vol. 47, pp. 2091-2094, July 2001.

[11] C. V. Chong and V. Tarokh, "A simple encodable/decodable OFDM QPSK code with low peak-to-mean envelope power ratio," IEEE Trans. Inform. Theory, vol. 47, pp. 3025-3029, Nov. 2001.

[12] K. G. Paterson and V. Tarokh, "On the existence and construction of good codes with low peak-to-average power ratio," IEEE Trans. Inform. Theory, vol. 46, pp. 1974-1987, Sept. 2000.

[13] M. Sharif and B. H. Khalaj, "Peak-to-mean envelope power ratio of oversampled OFDM signals: an analytical approach," in Proc. IEEE Int. Conf. Communications, vol. 5, Helsinki, Finland, June 2001, pp. 1476-1480.

[14] A. Zygmund, Trigonometric Series, 2nd ed. Cambridge, U. K.: Cambridge Univ. Press, 1968, vol. 2.

[15] H. Ochiai and H. Imai, "On the distribution of the peak to average power ratio in OFDM signals," IEEE Trans. Commun., vol. 49, pp. 282-289, Feb. 2001.

[16] D. Wulich, N. Dinur, and A. Glinowiecki, "Level clipped high-order OFDM,” IEEE Trans. Commun., vol. 48, pp. 928-930, June 2000.

[17] S. Shepherd, J. Orriss, and S. Barton, "Asymptotic limits in peak envelope power reduction by redundant coding in orthogonal frequency-division multiplexing," IEEE Trans. Commun., vol. 46, pp. 5-10, Jan. 1998. 


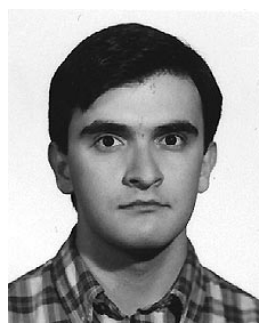

Masoud Sharif (S'99) was born in 1977. He received the B.Sc. (with honor) and M.Sc. degrees in electrical engineering from Sharif University of Technology, Tehran, Iran, in 1999 and 2001, respectively. He is currently working toward the Ph.D. degree in the Department of Electrical Engineering, California Institute of Technology, Pasadena, CA.

His research interests include information theory, wireless communications, and signal processing.

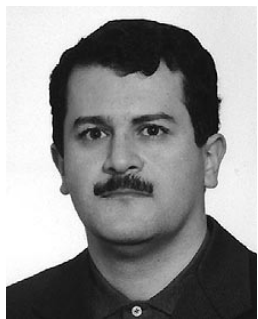

Mohammad Gharavi-Alkhansari (M'00) received the $\mathrm{Ph} . \mathrm{D}$. degree in electrical engineering from the University of Illinois at Urbana-Champaign, Urbana, in 1997.

From 1997 to 1998, he was a Postdoctoral Research Associate at the Beckman Institute for Advanced Science and Technology, University of Illinois at Urbana-Champaign. From 1998 to 1999, he was a Visiting Professor at the University of Tehran, Tehran, Iran. Since 1999, he has been an Assistant Professor at Tarbiat Modarres University, Tehran, Iran. In 2002, he joined the Smart Antenna Research Team at the Department of Communication Systems, Gerhard Mercator University of Duisburg, Duisburg, Germany. He is currently a Visiting Assistant Professor at McMaster University, Hamilton, ON, Canada.

Dr. Gharavi-Alkhansari is a member of Tau Beta Pi and Sigma Xi.

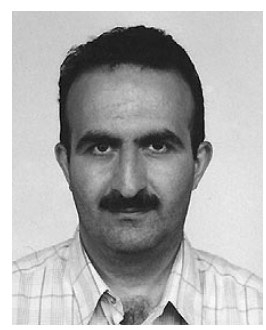

Babak H. Khalaj received the B.Sc. degree from Sharif University of Technology, Tehran, Iran, in 1989, and the M.Sc. and Ph.D. degrees from Stanford University, Stanford, CA, in 1992 and 1995, respectively, all in electrical engineering.

He joined KLA-Tencor in 1995 as a Senior Algorithm Designer working on advanced processing techniques for signal estimation. From 1996 to 1999 , he was with Advanced Fiber Communications and Ikanos Communications. Since then, he has been a Senior Consultant in the area of Data Communications. He was the Co-Editor of the Spectral Compatibility Standard Draft for the ANSI-T1E1 group from 1998 till 1999, and he is the author of a U.S. patent and many papers in signal processing and digital communications area. 